\title{
Nerve Fiber Analysis and Age-related Changes of the Human Mandibular Nerve
}

\author{
By \\ Kazuhiko OKA, Noboru GOTO, Naoko NONAKA, Jun GOTO and \\ Takamasa TSURUMI
}

Department of Anatomy, Showa University School of Medicine

- Received for Publication, January 29, 2001 -

\begin{abstract}
Key Words: Mandibular nerve, Myelinated nerve fibers, Trigeminal nerve, Axon, Human
Summary: We morphometrically analyzed nerve fibers of the human mandibular nerve with a discriminative staining method that makes it possible to separate nerve fibers into myelinated and unmyelinated fibers. We counted numbers and transverse areas of myelinated axons under the microscope using an on-line image-analyzer. This study revealed the morphometric changes which affect the human mandibular nerve during the aging process.
\end{abstract}

Up to now researches on the trigeminal nerve has been conducted using mainly on animals. We could find no morphometric study on the human trigeminal nerve or its branches except for several reports ${ }^{4,5)}$. However, except one concerning the diameters of the human myelinated trigeminal nerve fibers ${ }^{4}$. This was conducted on frozen sections, which adds a certain degree of unreliability to the redults. The aim of the present research was to reveal the composition of nerve fibers of the human mandibular nerve. We succeeded in counting and measuring the transverse area of myelinated axons with the help of an image-analyzer. This study revealed for the first time a morphometric change with age concerning the myelinated axons of the human mandibular nerve.

\section{Material and Methods}

Small sections of the mandiblular nerve were removed from 6 human cadavers (4 males and 2 females) with ages ranging from 59 to 95 years (average: 73.8 years). The causes of death showed no direct or indirect connection with the central and peripheral nervous systems. We adopted the same methods as in our previous researches regarding fixation, washing, dehydration, embedding, sectioning and staining ${ }^{1-3,6)}$. The sections were stained with Goto's modification of MassonGoldner's method. A morphometic analysis was conducted as follows: we counted the numbers of myelinated nerve fibers and measured the axonal areas with the help of a microscope equipped with a drawing tube (camera lucida) and an imageanalyzer.

\section{Results}

\section{Microscopic Findings}

There are two types of nerve fibers myelinated (in which appear dark-blue in color axons surrounded by a pink-colored myelin sheath) and unmyelinated (in which axons appear dark-blue surrounded by a light blue Schwann sheath).

\section{Morphometric Data}

\section{(1) Number of Myelinated Axons}

We estimated the total number of myelinated axons in the human mandibular nerve as being between 45 and 64 (average: 50) in a unit area of $70.30 \mu \mathrm{m}^{2}$. 


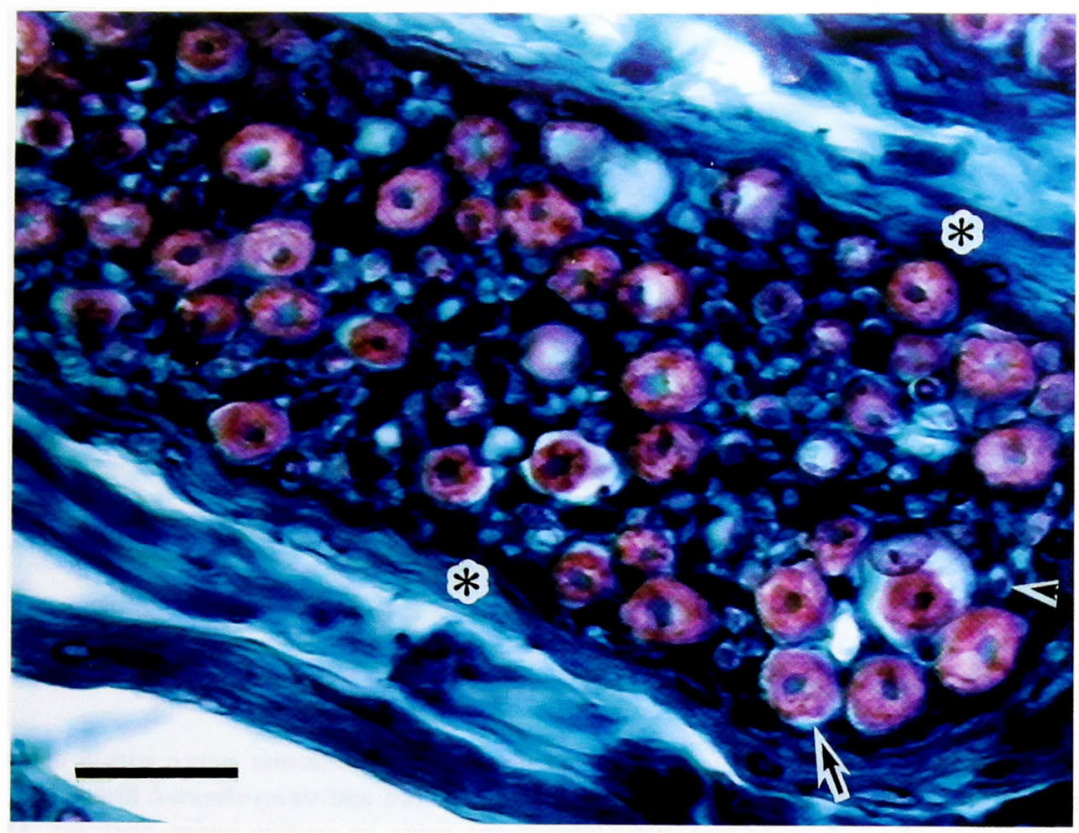

Fig. 1. A study of cross section of the human mandibular nerve under high-power view microscopy showed that the networks-shaped endneurium divides the nerves fibers into several separate bundles. arrow: myelinated nerve fiber; arrow head: unmyelinated nerve fiber; *: endneurium; Masson-Goldner-Goto stain, bar = $10 \mu \mathrm{m}$.

(2) Transverse Area of Myelinated Axons

The transverse area of myelinated axons in the human mandibular nerve ranged from 0.23 to 13.60 (average: 3.45$) \mu \mathrm{m}^{2}$. Scatter diagrams between area and perimeter of axons for every nerve showed a similar negative regression line. The average transverse area of myelinated axons was larger in the young individuals, than in the older group.

(3) Correlation between Morphometic Data and Aging

A scatter diagram with regression analysis between the average transverse area and age revealed that the average area of myelinated axons in the transverse section decreased with age $(r=-0.9460$; Fig. 2).

\section{Discussion}

The key element of this study is our methodology, which includes fixation, embedding, staining and morphometry. In all our reports ${ }^{1-3,6)}$, we have tried to reduce the shrinkage ratio of sections to the minimum ( $10 \pm 0 \%$ in length) by embedding in nitrocellulose after secondary fixation with a mixture solution of potassium chromate and potassium

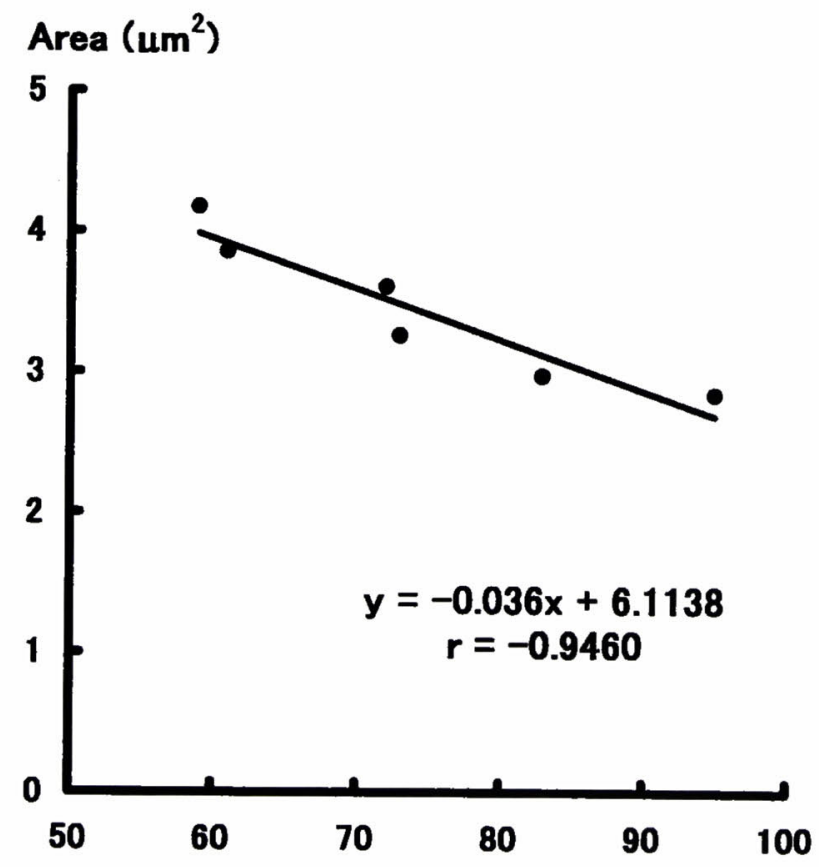

Fig. 2. Scatter diagram with regression line between axonal transverse area and age. 
dichromate. We concluded that Goto's modification of Masson-Goldner's method is the best staining method at present for the morphometric evaluation of nerve fibers ${ }^{1-3,6)}$. Adopting this method, we could easily distinguish axons from other tissue elements. In this paper, we could easily divide nerve fibers of the human mandibular nerve into myelinated and unmyelinated. Myelinated axons appeared blue in color and were surrounded by a thick pink myelin sheath, while unmylinated axons appeared also blue but surrounded by a thin light-blue Schwann sheath. The axons of myelinated nerve fibers, were larger than those of unmyelinated nerve fibers, as were the nerve fibers themselves. Up to now, there have been no reports on the analysis of axonal sizes. The negative correlation between transvers area and age $(\mathrm{r}=-0.9460)$ which we found during our analysis of myelinated axons proved that the axonal sizes of myelinated nerve fibers definitely decrease during the aging process ${ }^{6}$. . This fact could explain the slowing down of the conduction time of the nerve which appears during the aging process.

\section{References}

1) Goto J, Goto N, Ezure H, Nonaka N and Ma X. Morphogical differentation of nerve fibers: Central, peripheral, myelinated and unmyelinated. Okajimas Folia Anat Jpn (in press).

2) Goto N. Discriminative staining methods for the nervous system: Luxol fast blue-periodic acid-Schiff-hematoxylin triple stain and subsidiary attaining methods. Stain Technol 1987; 62:305-315.

3) Tang W, Goto N, Tanaka J and Otsuka N. Myelinated nerve fiber analysis of the human small splanchnic nerve. Okajimas Folia Anat Jpn 1997; 74:93-98.

4) Toyoda $Y$. Nerve fiber analysis of the human trigeminal nerve. Igaku kenkiyuu 1963; 37:599-607. (in Japanese)

5) Pennisi E, Cruccu G, Manfredi M and Palladini G. Histometric study of myelinated fibers in the human trigeminal nerve. J Neurol Sci 1991; 105:22-28.

6) Yanagisawa K, Goto N, Kimura T and Tanaka J. Nerve fiber analysis on aging process of deep peroneal nerve in man J Showa Med Soc 1994; 54:249-254. (in Japanese) 